\title{
A Modified Fast Approximated Power Iteration Subspace Tracking Method for Space-Time Adaptive Processing
}

\author{
Yang Zhiwei, ${ }^{1}$ He Shun, ${ }^{1,2}$ Liao Guisheng, ${ }^{1}$ and Ouyang Shan ${ }^{3}$ \\ ${ }^{1}$ National Laboratory of Radar Signal Processing, Xidian University, Xi'an 710071, China \\ ${ }^{2}$ Communication and Information Engineering Collage, Xi'an University of Science and Technology, Xi'an 710054, China \\ ${ }^{3}$ Department of Communication and Information Engineering, Guilin University of Electronic Technology, Guilin 541004, China
}

Correspondence should be addressed to Yang Zhiwei, zwyang@mail.xidian.edu.cn

Received 3 August 2010; Revised 22 November 2010; Accepted 16 December 2010

Academic Editor: Tamal Bose

Copyright (C) 2010 Yang Zhiwei et al. This is an open access article distributed under the Creative Commons Attribution License, which permits unrestricted use, distribution, and reproduction in any medium, provided the original work is properly cited.

\begin{abstract}
We propose a subspace-tracking-based space-time adaptive processing technique for airborne radar applications. By applying a modified approximated power iteration subspace tracing algorithm, the principal subspace in which the clutter-plus-interference reside is estimated. Therefore, the moving targets are detected by projecting the data on the minor subspace which is orthogonal to the principal subspace. The proposed approach overcomes the shortcomings of the existing methods and has satisfactory performance. Simulation results confirm that the performance improvement is achieved at very small secondary sample support, a feature that is particularly attractive for applications in heterogeneous environments.
\end{abstract}

\section{Introduction}

The primary goal of airborne ground moving target indicator (GMTI) radar is to detect and estimate the physical location of moving targets. Therefore, the clutter mitigation approach, that is, space-time adaptive processing (STAP) technique, must be employed to separate moving targets from stationary clutter. In essence, STAP combines spatial and temporal degrees of freedom to design a bidimension filter. It is recognized as central to effectively eliminate interference since the publication of Brennan and Reed [1], and the basic theory has been well developed in [2-5].

The optimal STAP processor derives a data-dependent weighting vector which can offer a significant increasing in output signal-to-interference-plus-noise ratio (SINR). However, this calculation requires the knowledge of the space-time covariance matrix at the cell under test (CUT). Two major reasons that have restrained its application in practice are the high computational cost and the substantial amount of stationary sample support (also called secondary data). These issues have motivated the development of suboptimal methodology such as reduced-rank STAP [611] and multichannel parametric STAP [12-15]. A recent overview of these algorithms can be found in [16].
Moreover, the STAP algorithm which is based on the use of subspace projection has been shown to be an attractive approach for rank reduction and can achieve good performance with very little sample support, since only the principal subspace needs to be estimated. The estimation of the principal subspace is commonly based on the traditional eigenvalue decomposition (EVD) or singular value decomposition (SVD). However, the main drawback of these decomposition approaches is that they are inherently computationally expensive. Therefore, a large number of algorithms have been proposed for performing the fast subspace tracking task. See [17-19] and references therein for some early work and $[20,21]$ for samples of more recent work.

We present here a new post-Doppler subspace-based STAP approach which is performed in two stages. The first step is used to recursively calculate the principal subspace. Once the signal subspace has been calculated, the adaptive weight vector can be achieved with the use of subspace projection in the last stage. This algorithm presents several advantages such as the following: an orthonormal subspace basis is computed at each updating step, the computational cost can be significantly reduced, the dimension of the dominant subspace can be adaptively determined, and 
convergence comparable with subspace estimation using the EVD can be achieved.

The paper is organized as follows. In Section 2, the subspace-based STAP algorithm and the dominant subspace tracking method are briefly introduced. In Section 3, our modified subspace tracking method based on the postDoppler adaptive architecture is developed. In Section 4, the performance of this method is compared to that of several subspace-based STAP algorithms among which are EVD, FAPI [22], and OPAST [23]. Finally, the main conclusions of this paper are summarized in Section 5.

\section{Problem Description}

2.1. Signal Model. The radar transmitter emits a pulse and collects samples of the received signal until the next pulse is emitted. Each of these samples corresponding to a given range is commonly referred to as "range bin". For each range bin, the data at the output of the array is arranged into a vector $\mathbf{x}$ of length $M \cdot N$, where $N$ is the number of pulses per coherent processing interval (CPI) and $M$ is the number of antennas. This space-time data can be more compactly written as

$$
\begin{gathered}
H_{0}: \mathbf{x}\left(r_{0}\right)=\sum_{i=1}^{\mathrm{Nc}} \gamma_{c}^{i} \cdot \mathbf{a}_{\mathrm{clu}}^{\mathbf{i}}+\mathbf{J}+\mathbf{n}, \\
H_{1}: \mathbf{x}\left(r_{0}\right)=\sum_{i=1}^{\mathrm{Nc}} \gamma_{c}^{i} \cdot \mathbf{a}_{\mathrm{clu}}^{\mathbf{i}}+\mathbf{S}+\mathbf{J}+\mathbf{n},
\end{gathered}
$$

in which $H_{0}$ is the null hypothesis indicating moving target absence, while $H_{1}$ is the alternative hypothesis indicating moving target presence. $\mathbf{J}$ represents the contribution of the jammers and $\mathbf{n}$ the additive thermal noise. Nc is the number of clutter patches considered in range bin $r_{0} . \gamma_{c}^{i}$ is the amplitude, and $\mathbf{a}_{\text {clu }}^{\mathbf{i}}=\mathbf{a}_{\text {clu }}^{\mathbf{s}}\left(f_{s}^{i}\right) \otimes \mathbf{a}_{\text {clu }}^{\mathbf{t}}\left(f_{d}^{i}\right)$ is the spacetime steering vector associated with the $i$ th clutter patch. $f_{s}^{i}$ represents the spatial frequency and $f_{d}^{i}$ the Doppler frequency. $\mathbf{a}_{\mathbf{c l u}}^{\mathbf{s}}\left(f_{s}^{i}\right)$ represents the spatial steering vector and $\mathbf{a}_{\text {clu }}^{\mathbf{t}}\left(f_{d}^{i}\right)$ the temporal steering vector. “ $\otimes$ " denotes the Kronecker tensor product. $\mathbf{S}=\gamma_{t} \cdot \mathbf{a}_{\mathrm{tar}}$ is the moving target term, where $\gamma_{t}$ and $\mathbf{a}_{\text {tar }}$ denote the target amplitude and the target space-time steering vector, respectively.

2.2. Subspace-Based STAP Algorithm. The weight vector of the linearly constrained minimum variance (LCMV) is given by

$$
\mathbf{w}_{\mathbf{o p t}}=\mathbf{R}_{H_{0}}^{-1}\left(r_{0}\right) \mathbf{a}_{\mathbf{t a r}},
$$

where $\mathbf{R}_{H_{0}}\left(r_{0}\right)$ is the space-time covariance matrix under null hypothesis, and “ $(\cdot)^{\mathrm{H}}$ ” is complex conjugate transpose. In practice, $\mathbf{R}_{H_{0}}\left(r_{0}\right)$ is unknown and needs to be estimated from the training data, in which case

$$
\mathbf{w}_{\mathbf{o p t}}=\widehat{\mathbf{R}}_{H_{0}}^{-1}\left(r_{0}\right) \mathbf{a}_{\mathbf{t a r}},
$$

where $\hat{\mathbf{R}}_{H_{0}}\left(r_{0}\right)$ is the sample covariance matrix which can be formed from a sufficiently larger number $L$ of range bins adjacent to the range $r_{0}$. It is also referred to as the sample matrix inversion (SMI) algorithm.

Note that the clutter-plus-jammer component exists in a relatively low dimensional subspace of the $M \cdot N$-dimensional space of the space-time data. Let $\hat{\mathbf{R}}_{H_{0}}\left(r_{0}\right)=\mathbf{U} \boldsymbol{\Lambda} \mathbf{U}^{\mathrm{H}}$ be the eigenvalue decomposition of the sample covariance matrix. Let $\mathbf{E}_{\boldsymbol{S}}$ be the matrix consisting of the columns of $\mathbf{U}$ associated with the largest $p$ eigenvalues. Then, $\mathbf{E}_{\mathbf{S}}$ is the rank- $p$ approximation to the clutter-plus-jammer subspace, which we call the principal subspace or the signal subspace. The remaining columns of $\mathbf{U}$ span a subspace orthogonal to $\mathbf{E}_{\mathrm{S}}$, which we denote by $\mathbf{E}_{\mathrm{N}}$ and call the minor subspace or the noise subspace. Therefore, the weight vector can be rewritten as

$$
\mathbf{w}_{\mathbf{o p t}}=\left(\mathbf{E}_{\mathrm{S}} \boldsymbol{\Lambda}_{\mathrm{S}}^{-1} \mathbf{E}_{\mathrm{S}}^{\mathrm{H}}+\mathbf{E}_{\mathrm{N}} \boldsymbol{\Lambda}_{\mathrm{N}}^{-1} \mathbf{E}_{\mathrm{N}}^{\mathrm{H}}\right) \mathbf{a}_{\mathrm{tar}}
$$

where $\Lambda_{\mathbf{S}}$ is the diagonal matrix consisting of the largest $p$ eigenvalues and $\Lambda_{\mathrm{N}}$ consisting of the smallest $M \cdot N-p$ eigenvalues.

Since we chose $p$ so that $\zeta=\operatorname{tr}\left(\Lambda_{\mathbf{S}}\right) / \operatorname{tr}(\boldsymbol{\Lambda})$, it can be very close to unity where " $\operatorname{tr}(\cdot)$ " is the trace operator. Moreover, each of these smallest $M \cdot N-p$ eigenvalues is approximately identical. Therefore, the subspace-based weight vector in the so-called principal component canceller is given by,

$$
\mathbf{w}_{\mathrm{SP}}=\left(\mathbf{I}_{M \cdot N}-\mathbf{E}_{\mathrm{S}} \mathbf{E}_{\mathrm{S}}^{\mathrm{H}}\right) \mathbf{a}_{\mathrm{tar}}=\mathbf{E}_{\mathrm{N}} \mathbf{E}_{\mathbf{N}}^{\mathrm{H}} \mathbf{a}_{\mathrm{tar}},
$$

where $\mathbf{I}_{M \cdot N}$ denotes an identity matrix of dimension $M \cdot N$.

Thanks to the rank-reduction nature of the space-time correlation matrix, the subspace-based methods are known to converge faster than SMI with very little sample support. Popularly, the size of the secondary data sample set of the principal component canceller approach can be reduced to $L \geq 2 \cdot p$. However, the algorithm is computationally costly, since the very expensive EVD of space-time covariance matrix, involving $O\left((M \cdot N)^{3}\right)$ complex operations, is still included.

2.3. Introducing Subspace Tracking into STAP Algorithm. The complexity of the dominant subspace estimation with EVD procedure can be reduced by adopting subspace tracking method. These subspace trackers are based on the minimization of the mean square error according to the following cost function:

$$
J\left(\mathbf{U}_{\mathbf{S}}\right)=\mathbf{E}\left\langle\left\|\mathbf{x}\left(r_{k}\right)-\mathbf{U}_{\mathbf{S}} \mathbf{U}_{\mathbf{S}}^{\mathrm{H}} \mathbf{x}\left(r_{k}\right)\right\|^{2}\right\rangle,
$$

where $\mathbf{x}\left(r_{k}\right)$ denotes the data vector observed on secondary range bins, and $\mathbf{U}_{\mathbf{S}} \in \mathbf{C}^{M \cdot N \times p}$ is the $p$-dimensional dominant subspace which is spanned by the correlation matrix. The dominant subspace can be reliably estimated, since this cost function has a single global minimum which is attained for $\mathbf{U}_{\mathbf{S}}=\mathbf{E}_{\mathbf{S}} \mathbf{Q}$, where $\mathbf{Q} \in \mathbf{C}^{p \times p}$ and $\mathbf{Q}^{\mathrm{H}} \mathbf{Q}=\mathbf{Q} \mathbf{Q}^{\mathrm{H}}=\mathbf{I}_{\mathbf{p}}$.

In spite of the fact that different subspace tracking methods can be derived, here, we are more particularly interested in the FAPI algorithm [22], which is a good candidate owing to its efficiency and robustness. To provide 
a better estimation of the dominant subspace, the cost function in FAPI algorithm is replaced by

$$
J\left(\mathbf{U}_{\mathbf{S}}(L)\right)=\sum_{k=1}^{L} \beta^{L-k}\left\|\mathbf{x}\left(r_{k}\right)-\mathbf{U}_{\mathbf{S}}(L) \mathbf{U}_{\mathbf{S}}^{\mathrm{H}}(L) \mathbf{x}\left(r_{k}\right)\right\|^{2},
$$

in which $0<\beta \leq 1$ is the forgetting factor, and $\mathbf{U}_{\mathbf{S}}$ is replaced by $\mathbf{U}_{\mathbf{S}}(L)$.

The key of this algorithm is the assumption of $\mathbf{U}_{\mathbf{S}}(L-1)$ exactly spans the $p$-dimensional dominant subspace of the correlation matrix, Therefore, $\mathbf{U}_{\mathbf{S}}(L)$ and $\mathbf{U}_{\mathbf{S}}(L-1)$ are two orthonormal matrices spanning the identical range space, that is, $\mathbf{U}_{\mathbf{S}}(L)=\mathbf{U}_{\mathbf{S}}(L-1) \Theta(L)$, where $\boldsymbol{\Theta}(L)=\mathbf{U}_{\mathbf{S}}^{\mathrm{H}}(L-$ 1) $\mathbf{U}_{\mathrm{S}}(L)$ is a $p \times p$ orthonormal matrix. The derivation of the FAPI algorithm is not given here, since it can be found in detail in [22]. Note only that a particular selection for the $\boldsymbol{\Theta}(L)$ matrix can reduce the computational cost dramatically, and the dominant subspace is obtained from the power iteration and projection approximation method.

Once the principal subspace was estimated, the subspacebased weight vector is then

$$
\mathbf{w}_{\mathbf{S P}}(L)=\left(\mathbf{I}_{M \cdot N}-\mathbf{U}_{\mathbf{S}}(L) \mathbf{U}_{\mathbf{S}}^{\mathrm{H}}(L)\right) \mathbf{a}_{\mathbf{t a r}} .
$$

\section{Modification of FAPI for Post-Doppler STAP}

3.1. Motivation of the Proposed Algorithm. Two factors affect the direct use of FAPI for the fully adaptive STAP application. First, since the FAPI algorithm updates all dominant eigenvectors per iteration, it propagates the error of eigenbasis derivation. This error propagation consequently decreases the convergence rate of the dominant subspace. Second, either adopting subspace methods or not, all the characteristics of the received data, such as clutter locus, are not taken into consideration in fully adaptive STAP.

In fact, an improved performance of convergence rate can be achieved if the aforementioned error propagation effect can be avoided. Moreover, using the partially adaptive architecture, the performance of SINR loss can be improved. Note that the post-Doppler approaches, which provides a good tradeoff between detection performance and computational burden, are indeed the most popular among others. That is why only a modified FAPI method for post-Doppler STAP will be considered in here.

3.2. Description of the Proposed Algorithm. Let $q$ denote the number of adjacent Doppler bins. Therefore, for a given range gate $r_{l}$ and Doppler frequency $f_{d}^{n}$, the $(q+1) M \times 1$ post-Doppler data vector can be written as

$$
\mathbf{x}_{\mathbf{J}}\left(r_{l}, f_{d}^{n}\right)=\left[\begin{array}{llll}
\mathbf{x}\left(r_{l}, f_{d}^{n}\right)^{\mathrm{T}} & \mathbf{x}\left(r_{l}, f_{d}^{n_{1}}\right)^{\mathrm{T}} \cdots & \mathbf{x}\left(r_{l}, f_{d}^{n_{q}}\right)^{\mathrm{T}}
\end{array}\right]^{\mathrm{T}},
$$

in which $\left\{f_{d}^{n_{j}}\right\}_{j=1}^{q}$ is a set of adjacent Doppler bins around $f_{d}^{n}$, and $(\cdot)^{\mathrm{T}}$ denotes transpose. The typical structure for obtaining $\mathbf{x}\left(r_{l}, f_{d}^{n_{j}}\right)$ is presented in Figure 1 .

Then, instead of updating all dominant eigenvectors per iteration, we will estimate principal eigenvectors sequentially

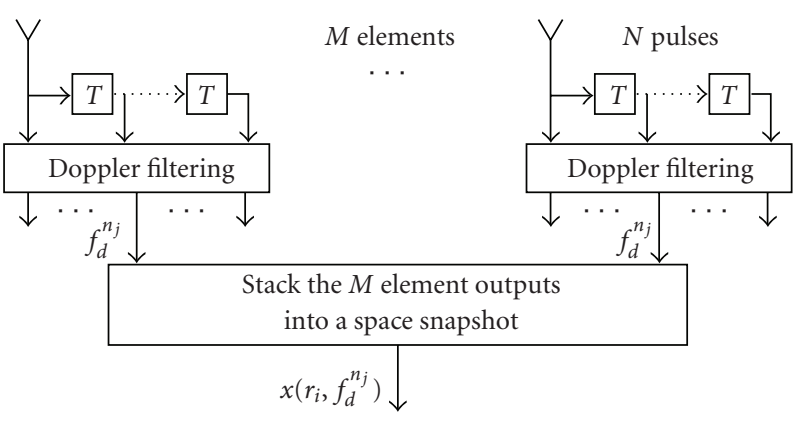

FIGURE 1: Representations of space snapshot for given range gate $r_{l}$ and Doppler frequency $f_{d}^{n_{j}}$.

utilizing all training data. Let $\mathbf{v}_{1}(l)$ be the most dominant eigenvector, and the reciprocal of $z_{1}(l)$ is the corresponding eigenvalue at the $l$ th iteration. The estimation procedure based on FAPI is presented as follows:

Initialization : $\mathbf{v}_{1}(0)=\left[\begin{array}{c}1 \\ \mathbf{0}_{((q+1) M-1) \times 1}\end{array}\right] z_{1}(0)=1$,

for $l=1$ to $L$ post-Doppler snapshots do

$$
\begin{aligned}
& y(l)=\mathbf{v}_{1}(l-1)^{\mathrm{H}} \mathbf{x}_{\mathbf{J}}\left(r_{l}, f_{d}^{n}\right), \\
& g(l)=\frac{z_{1}(l-1) y(l)}{\left(\beta+z_{1}(l-1)\|y(l)\|^{2}\right)} \\
& \varepsilon^{2}(l)=\left\|\mathbf{x}_{\mathbf{J}}\left(r_{l}, f_{d}^{n}\right)\right\|^{2}-\|y(l)\|^{2} \\
& \tau(l)=\frac{\varepsilon^{2}(l)}{\left(1+\varepsilon^{2}(l)\|g(l)\|^{2}+\sqrt{1+\varepsilon^{2}(l)\|g(l)\|^{2}}\right)} \\
& \eta(l)=1-\tau(l)\|g(l)\|^{2}, \\
& y^{\prime}(l)=\left(\eta(l)+\frac{\tau(l) z_{1}(l-1)}{\left(\beta+z_{1}(l-1)\|y(l)\|^{2}\right)}\right) y(l), \\
& \varsigma(l)=\frac{\tau(l)}{\eta(l)}\left(1-y^{\prime}(l)^{*} g(l)\right) z_{1}(l-1) g(l), \\
& z_{1}(l)=\frac{\left(z_{1}(l-1)-g(l) z_{1}(l-1) y^{\prime}(l)^{*}+\varsigma(l) g(l)^{*}\right)}{\beta}, \\
& \mathbf{v}_{1}(l)=\mathbf{v}_{1}(l-1)+g(l)^{*}\left(\eta(l) \mathbf{x}_{\mathbf{J}}\left(r_{l}, f_{d}^{n}\right)-y^{\prime}(l) \mathbf{v}_{1}(l-1)\right),
\end{aligned}
$$

End

where $(\cdot)^{*}$ is complex conjugate. 
Once the first eigenvector $\mathbf{v}_{1}(L)$ has been achieved, the clearing operation removes the projection of each training snapshot onto $\mathbf{v}_{1}(L)$ can be written as

$$
\begin{aligned}
& \text { for } l=1 \text { to } L \text { post-Doppler snapshots do } \\
& \qquad \begin{array}{l}
y(l)=\mathbf{v}_{1}(L)^{\mathrm{H}} \mathbf{x}_{\mathbf{J}}\left(r_{l}, f_{d}^{n}\right), \\
\mathbf{x}_{\mathbf{J}}\left(r_{l}, f_{d}^{n}\right)=\mathbf{x}_{\mathbf{J}}\left(r_{l}, f_{d}^{n}\right)-\mathbf{v}_{1}(L) y(l) .
\end{array}
\end{aligned}
$$

End

Since all training data are projected onto the same eigenbasis, the error propagation cased by the eigenbasis deviation is avoided. Now, the second dominant eigenvector $\mathbf{v}_{2}(L)$ is the most dominant one in $\left\{\mathbf{x}_{\mathbf{J}}\left(r_{l}, f_{d}^{n}\right)\right\}_{l=1}^{L}$ and can be obtained in the same way as $\mathbf{v}_{1}(L)$ described in (10). Thus, all desired eigenvectors can be worked out sequentially, which is referred to as m-FAPI algorithm and described as follows:

for $p=1$ to $P$ principal eigenvectors do

$$
\begin{aligned}
& \left\{\mathbf{x}_{\mathbf{J}}\left(r_{l}, f_{d}^{n}\right)\right\}_{l=1}^{L}, \\
& \text { estimate } \mathbf{v}_{\mathbf{p}}(L) \text { and } z_{p}(L) \\
& \operatorname{Orth}\left(\mathbf{v}_{1}(L) \cdots \mathbf{v}_{\mathbf{p}}(L)\right), \\
& \text { for } l=1 \text { to } L \text { post-Doppler snapshots do } \\
& \qquad y(l)=\mathbf{v}_{\mathbf{p}}(L)^{\mathrm{H}} \mathbf{x}_{\mathbf{J}}\left(r_{l}, f_{d}^{n}\right), \\
& \quad \mathbf{x}_{\mathbf{J}}\left(r_{l}, f_{d}^{n}\right)=\mathbf{x}_{\mathbf{J}}\left(r_{l}, f_{d}^{n}\right)-\mathbf{v}_{\mathbf{p}}(L) y(l),
\end{aligned}
$$

End

End

where $\operatorname{Orth}\left(\mathbf{v}_{1}(L) \cdots \mathbf{v}_{\mathbf{p}}(L)\right)$ denotes the operator in which an orthonormal basis for the range of $\left[\begin{array}{lll}\mathbf{v}_{1}(L) & \cdots & \mathbf{v}_{\mathbf{p}}(L)\end{array}\right]$ can be obtained.

Remark 1. We clarify that our goal here is not to justify whether the partially adaptive STAP approaches with FAPI are of appropriateness or not. An answer to the question can be found in [22], where it is shown that the FAPI algorithm is very powerful and efficient in tracking the principal subspace. Since the m-FAPI algorithm extracts principal eigenvectors sequentially utilizing all training data, the error propagation caused by updating all dominant eigenvectors per iteration will be avoided. The comparison between the subspace estimation error with m-FAPI and that with FAPI will be investigated in Section 4 .

Remark 2. Perhaps the biggest difficulty associated with adopting a principal subspace tracker for STAP is determining what subspace dimension results in the best performance. The assumption that the subspace dimension is known is only used to simplify our presentation. In practice, adopting the well-known selection techniques, such as the
Akaike information criterion (AIC) [24] and the minimum description length- (MDL-) [25] based techniques, the subspace dimension can be adaptively determined with $\left\{z_{p}(L)\right\}_{p=1}^{P}$. Although robustness of the subspace dimension selection would be of value in real application, it is beyond the scope of the current paper.

Remark 3. We would like to point out that the orthonormality between the estimated eigenvectors cannot be guaranteed by adopting only the clearing operation due to a numerical stability problem and noise, especially when the dimension of the estimated subspace is larger than that of the true dominant subspace and/or the train data set is small. However, our m-FAPI algorithm can guarantee the orthonormality between the estimated eigenvectors at each iteration.

\section{Simulation Results}

In this section, two simulations are provided to illustrate the effectiveness of our proposed approach. Firstly, the performance of the subspace estimation is analyzed according to the following estimation error cost function:

$$
\begin{aligned}
& f_{\mathbf{U}(L)} \\
& =20 \log _{10}\left(\left\|\mathbf{P}_{\mathbf{U}(L)}-\mathbf{P}_{\mathbf{E}_{\mathbf{S}}}\right\|\right)+20 \log _{10}\left(\frac{\left\|\mathbf{U}(L)^{\mathrm{H}} \mathbf{U}(L)-\mathbf{I}_{\mathbf{P}}\right\|}{\left\|\mathbf{E}_{\mathbf{S}}^{\mathrm{H}} \mathbf{E}_{\mathbf{S}}-\mathbf{I}_{\mathbf{P}}\right\|}\right),
\end{aligned}
$$

where $\mathbf{P}_{\mathbf{U}(L)}=\mathbf{U}(L)\left(\mathbf{U}(L)^{\mathrm{H}} \mathbf{U}(L)\right)^{-1} \mathbf{U}(L)^{\mathrm{H}}$ and $\mathbf{P}_{\mathrm{E}_{\mathbf{S}}}=$ $\mathbf{E}_{\mathbf{S}}\left(\mathbf{E}_{\mathbf{S}}^{\mathrm{H}} \mathbf{E}_{\mathbf{S}}\right)^{-\mathbf{1}} \mathbf{E}_{\mathbf{S}}^{\mathrm{H}}$ denote the projection matrix that projects onto the column space of $\mathbf{U}(L)$ (obtained with the subspace tracker) and $\mathbf{E}_{S}$ (obtained via an exact eigenvalue decomposition), respectively. In this simulation, we use an eleven-element uniform linear array (ULA) with half wavelength interspacing and consider four uncorrelated signals coming from $\left[\begin{array}{llll}-20^{0} & 0^{0} & 15^{0} & 30^{0}\end{array}\right]$, that is, $M=11$ and $P=4$. For the sake of simplicity, we are assuming that the dimension of the dominant subspace is exactly known. The forgetting factor of the m-FAPI and the FAPI approach is $\beta=0.99$. This estimation error $f_{\mathrm{U}(L)}$ versus sample number is shown in Figure 2(a) with 1000 Monte Carlo trials, and the single-to-noise ratio (SNR) is given by $[20 \mathrm{~dB} \quad 20 \mathrm{~dB} \quad 20 \mathrm{~dB} 20 \mathrm{~dB}$ ]. It can be seen that the m-FAPI algorithm and the FAPI algorithm reach the almost same performance with the number of training data increasing when the tested signals have equal SNR, except at small sample support, where m-FAPI estimate better. It also can be noticed that the difference is much more distinct with the m-FAPI algorithm and the OPAST algorithm, which means that m-FAPI converges faster. A similar result is also given in Figure 2(b), where SNR is given by $[30 \mathrm{~dB} 20 \mathrm{~dB} 10 \mathrm{~dB} 20 \mathrm{~dB}]$. In both cases, the m-FAPI algorithm has best performance among these algorithms. Moreover, the computational cost of the mFAPI is lower $(O(L \cdot(q+1) M \cdot P)$, where $P$ is the rank of the clutter covariance matrix for post-Doppler processing) than the non subspace tracking algorithms under 

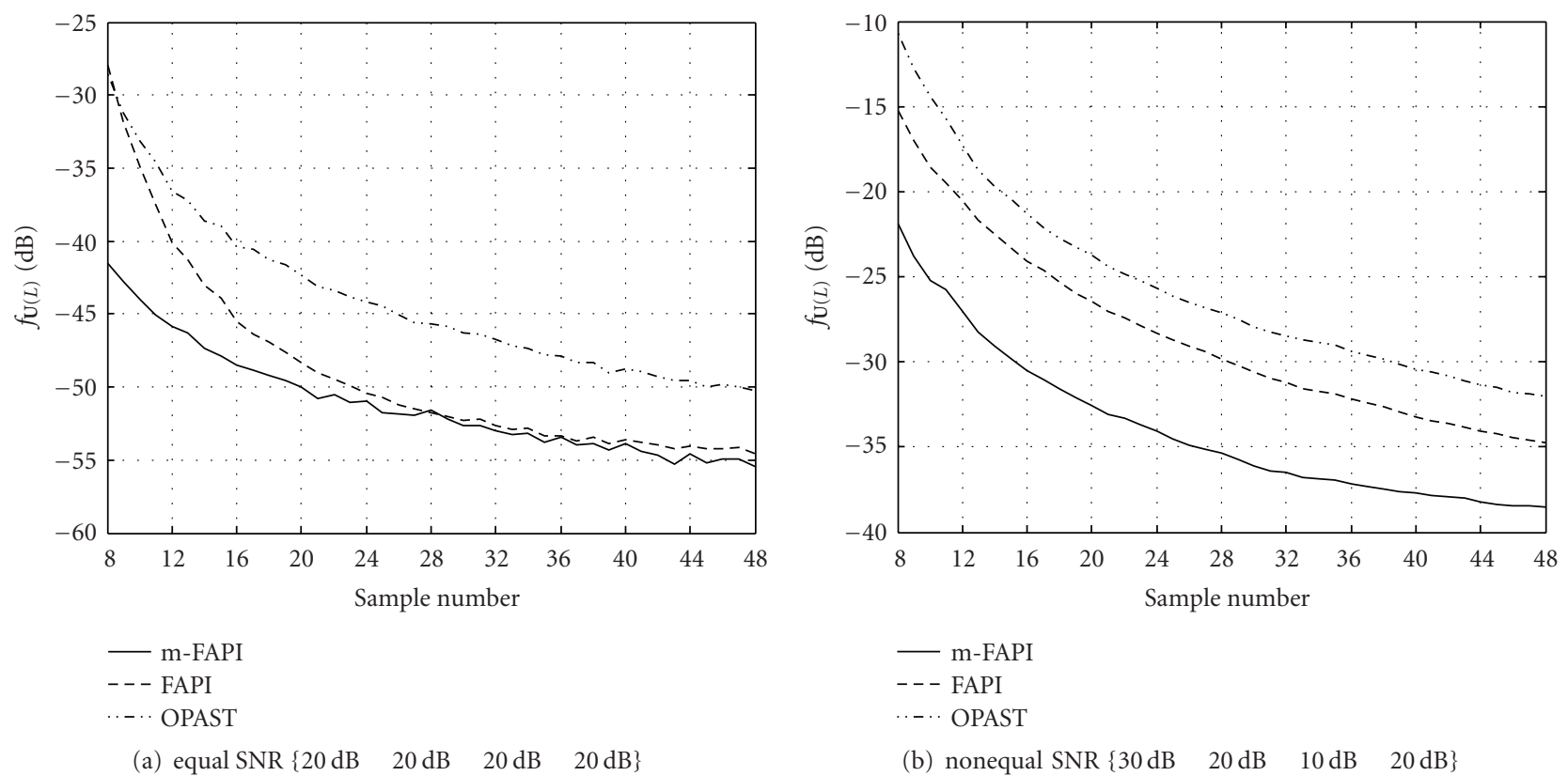

Figure 2: The estimation error $f_{\mathrm{U}(L)}$ versus sample number with $M=11$ and $P=4$.

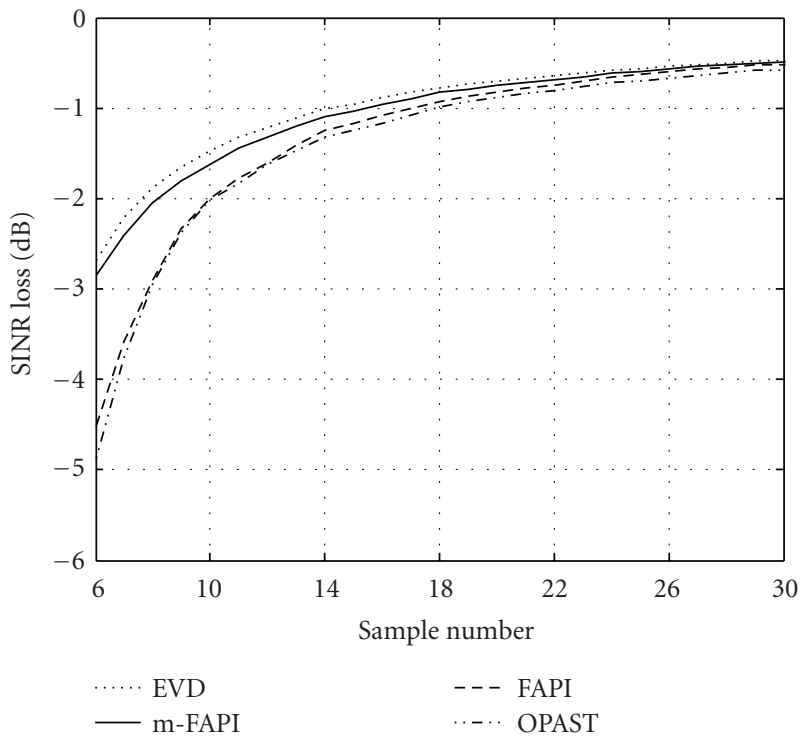

FIgURE 3: SINR loss versus sample number.

consideration $\left(O\left((L \cdot(q+1) M)^{3}\right)\right)$ for post-Doppler SMI and for $O\left((L \cdot M \cdot N)^{3}\right)$ for full-dimensional SMI.

Secondly, the performance of clutter cancellation is analyzed based on the SINR loss, which is defined as the loss in SINR compared with a matched filter in the presence of white noise only. We consider a side-looking airborne radar, operating at SS-band with an eleven-element ULA and using of $N=16$ pulses per CPI. Typical platform velocities are $120 \mathrm{~m} / \mathrm{s}$, and the aircraft flies $5 \mathrm{~km}$ above the observed surface. The clutter-to-noise ratio (CNR) is given by $\mathrm{CNR}=30 \mathrm{~dB}$, and the pulse repetition frequency (PRF)

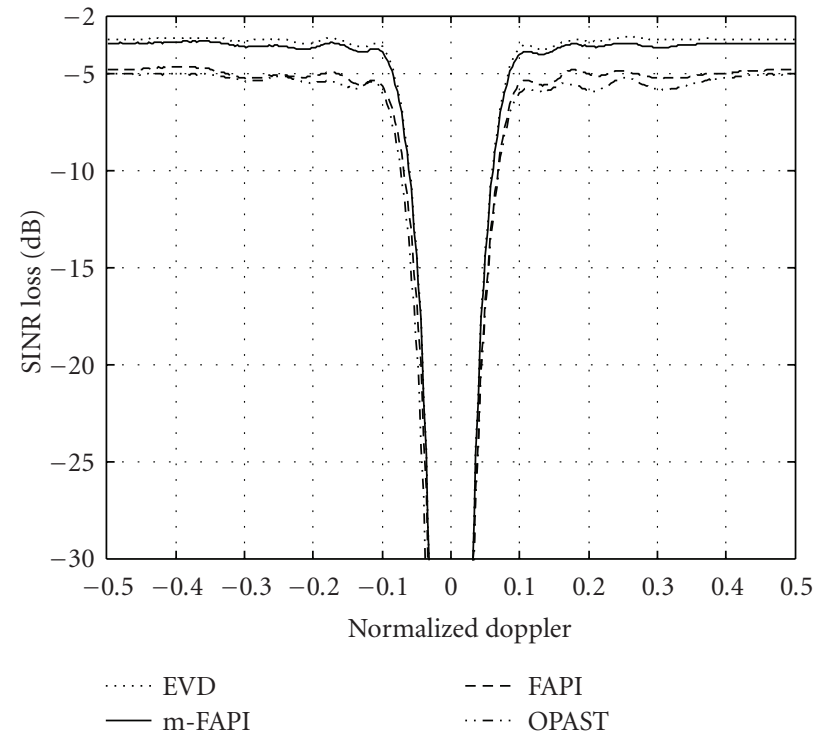

FIGURE 4: SINR loss versus normalized Doppler.

has been chosen to avoid both range and Doppler ambiguity in the main lobe. We apply the EFA-STAP technique [6] which adaptively combining all spatial channels with three adjacent Doppler channels. Figure 3 shows the adaptive SINR loss against sample number, where the target normalized Doppler frequency equals to 0.25 . As seen in the figure, all of these algorithm reach the almost same performance with the number of training data increasing. However, the converges rate of m-FAIP algorithm is much more rapidly than that of FAPI algorithm and OPAST algorithm, and the SINR loss of m-FAPI algorithm is quite close to that of EVD algorithm. 
As a finial comparison, Figure 4 provides the SINR loss as a function of the target normalized Doppler frequency, where the number of I.I.D training data has been selected as 6 . The deep null in the figure is caused by the location of the clutter ridge. It is obvious that our method leads to approximately $2 \mathrm{~dB}$ performance improvement over FAPI and OPAST and achieves a performance quite comparable with EVD method with very small secondary sample support. Similar to the first simulation, the results support the discussions in Section 3 and illustrate the efficiency of our proposed method.

\section{Conclusion}

STAP technique is the bidimensional adaptive processing in space and time which is employed for the purposes of clutter mitigation to enable the detection of slow moving targets. In this paper, a new method of partially adaptive STAP was proposed. The approach is based on the use of subspace projection, where the principal subspace has been tracked with m-FAPI algorithm. Both in the terms of estimation error and SINR loss, the method is proven able to outperform FAPI and OPAST algorithm. Furthermore, it is shown that m-FAPI algorithm converges quickly, and the method achieves clutter mitigation performance quite comparable with the EVD method. Finally, the proposed subspace tracking algorithm can be applied as the starting point of a real-time STAP processor, where the expensive EVD procedure in eigenvector computation can be avoided.

\section{Acknowledgments}

This work was supported by the National Natural Science Foundation of China (60901066), the New Teacher Foundation of Ministry of Education (20090203120006), and the Fundamental Research Funds for the Central University.

\section{References}

[1] L. E. Brennan and L. S. Reed, "Theory of adaptive radar," IEEE Transactions on Aerospace and Electronic Systems, vol. 9, no. 2, pp. 237-252, 1973.

[2] J. Ward, "Space-time adaptive processing for airborne radar systems," Tech. Rep. 1015, DTIC AD-A293032, Lincoln Laboratory, Massachusetts Institute of Technology, Lexington, Mass, USA, 1994.

[3] R. Klemm, Principles of Space-Time Adaptive Processing, Institute of Electrical Engineering, London, UK, 2002.

[4] J. R. Guerci, Space-Time Adaptive Processing for Radar, Artech House, Norwood, Mass, USA, 2003.

[5] W. L. Melvin, "A STAP overview," IEEE Aerospace and Electronic Systems Magazine, vol. 19, no. 1, pp. 19-35, 2004.

[6] R. C. DiPietro, "Extended factored space-time processing for airborne radar," in Proceedings of the 26th Asilomar Conference on Signals, Systems, and Computers, pp. 425-430, Pacific Grove, Calif, USA, October 1992.

[7] Z. Bao, G. Liao et al., "Adaptive spatial-temporal processing for airborne radars," Chinese Journal of Electronics, vol. 2, no. 1, pp. 1-7, 1993.

[8] H. Wang and L. Cai, "On adaptive spatial-temporal processing for airborne surveillance radar systems," IEEE Transactions on
Aerospace and Electronic Systems, vol. 30, no. 3, pp. 660-670, 1994.

[9] R. D. Brown, R. A. Schneible, M. C. Wicks, H. Wang, and Y. Zhang, "STAP for clutter suppression with sum and difference beams," IEEE Transactions on Aerospace and Electronic Systems, vol. 36, no. 2, pp. 634-646, 2000.

[10] J. R. Guerci, J. S. Goldstein, and I. S. Reed, "Optimal and adaptive reduced-rank STAP," IEEE Transactions on Aerospace and Electronic Systems, vol. 36, no. 2, pp. 647-663, 2000.

[11] R. C. de Lamare and R. Sampaio-Neto, "Adaptive reducedrank processing based on joint and iterative interpolation, decimation, and filtering," IEEE Transactions on Signal Processing, vol. 57, no. 7, pp. 2503-2514, 2009.

[12] J. R. Roman, M. Rangaswamy, D. W. Davis, Q. Zhang, B. Himed, and J. H. Michels, "Parametric adaptive matched filter for airborne radar applications," IEEE Transactions on Aerospace and Electronic Systems, vol. 36, no. 2, pp. 677-692, 2000.

[13] P. Parker and A. Swindlehurst, "Space-time autoregressive filtering for matched subspace STAP," IEEE Transactions on Aerospace and Electronic Systems, vol. 39, no. 2, pp. 510-520, 2003.

[14] K. J. Sohn, H. Li, and B. Himed, "Parametric Rao test for multichannel adaptive signal detection," IEEE Transactions on Aerospace and Electronic Systems, vol. 43, no. 3, pp. 920-933, 2007.

[15] K. J. Sohn, H. Li, and B. Himed, "Parametric GLRT for multichannel adaptive signal detection," IEEE Transactions on Signal Processing, vol. 55, no. 11, pp. 5351-5360, 2007.

[16] S. De Greve, P. Ries, F. D. Lapierre, and J. G. Verly, "Framework and taxonomy for radar space-time adaptive processing (STAP) methods," IEEE Transactions on Aerospace and Electronic Systems, vol. 43, no. 3, pp. 1084-1099, 2007.

[17] D. J. Rabideau, "Fast, rank adaptive subspace tracking and applications," IEEE Transactions on Signal Processing, vol. 44, no. 9, pp. 2229-2244, 1996.

[18] B. Yang, "Projection approximation subspace tracking," IEEE Transactions on Signal Processing, vol. 43, no. 1, pp. 95-107, 1995.

[19] P. Strobach, "Low-rank adaptive filters," IEEE Transactions on Signal Processing, vol. 44, no. 12, pp. 2932-2947, 1996.

[20] X. G. Doukopoulos and G. V. Moustakides, "Fast and stable subspace tracking," IEEE Transactions on Signal Processing, vol. 56, no. 4, pp. 1452-1465, 2008.

[21] R. Badeau, G. Richard, and B. David, "Fast and stable YAST algorithm for principal and minor subspace tracking," IEEE Transactions on Signal Processing, vol. 56, no. 8 I, pp. 34373446, 2008.

[22] R. Badeau, B. David, and G. Richard, "Fast approximated power iteration subspace tracking," IEEE Transactions on Signal Processing, vol. 53, no. 8, pp. 2931-2941, 2005.

[23] K. Abed-Meraim, A. Chkeif, and Y. Hua, "Fast orthonormal PAST algorithm," IEEE Signal Processing Letters, vol. 7, no. 3, pp. 60-62, 2000.

[24] H. Akaike, "A new look at the statistical model identification," IEEE Transactions on Automatic Control, vol. 19, no. 6, pp. 716-723, 1974.

[25] J. Rissanen, "Modeling by shortest data description," Automatica, vol. 14, no. 5, pp. 465-471, 1978. 

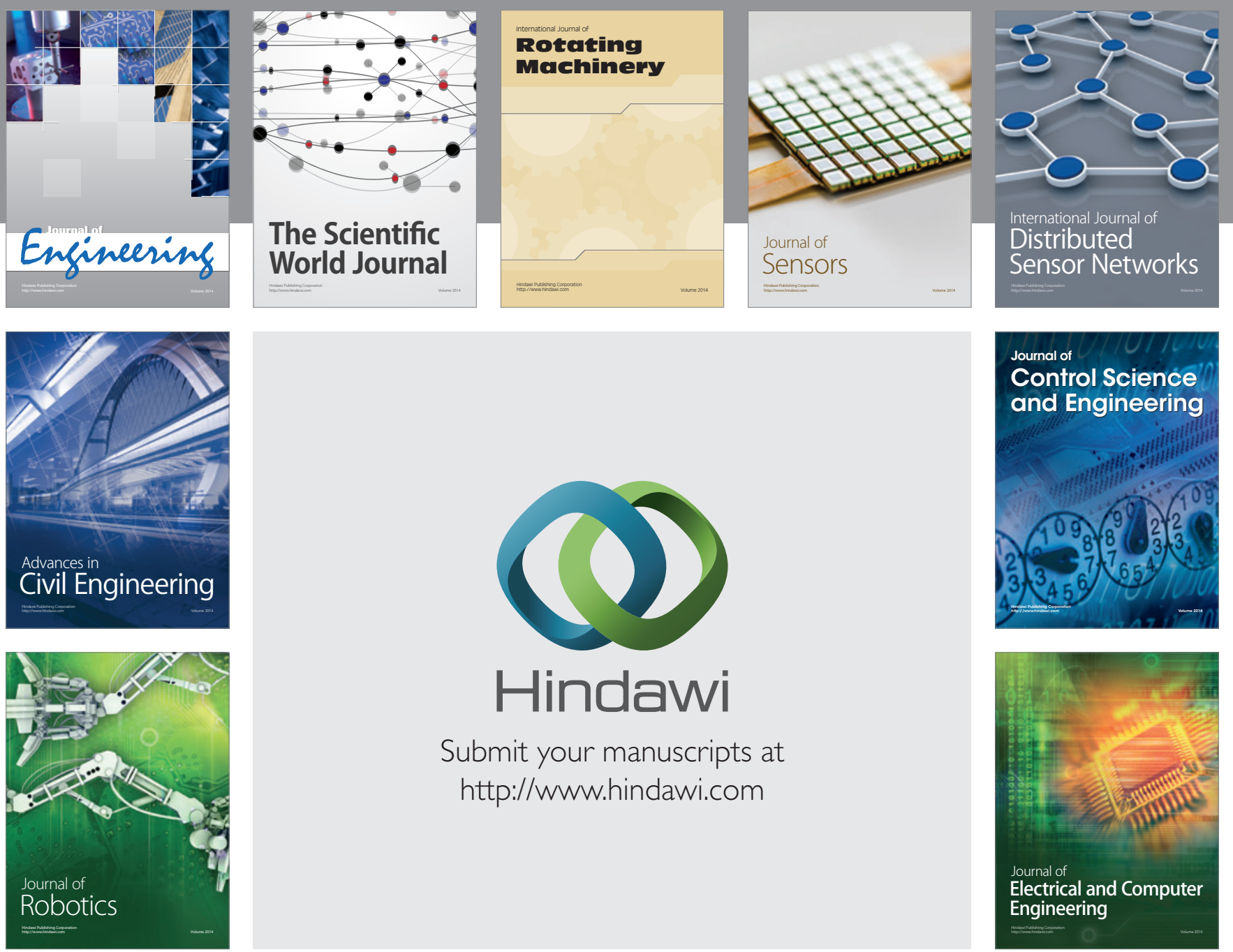

Submit your manuscripts at

http://www.hindawi.com
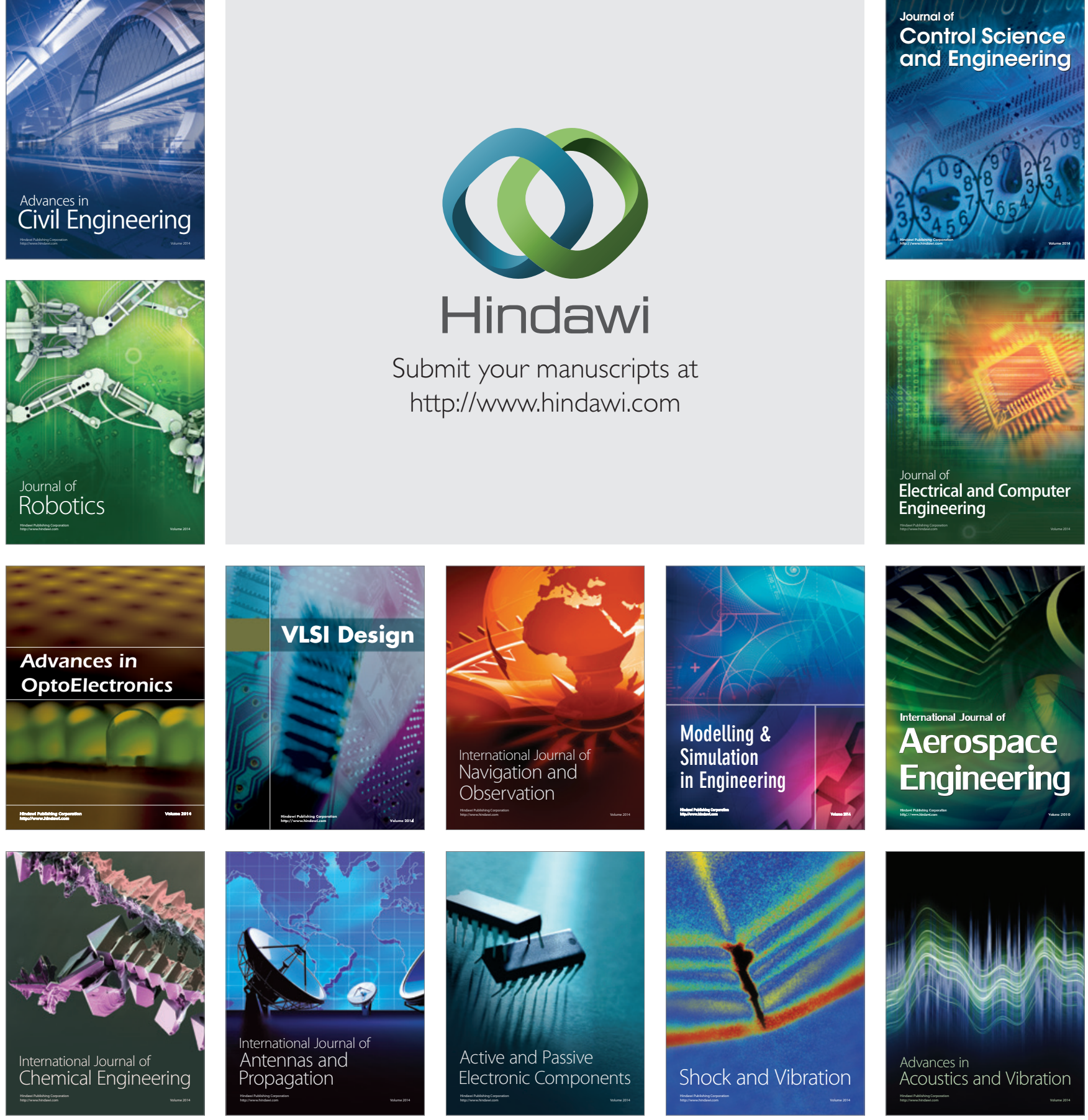